BULLETIN OF THE

AMERICAN MATHEMATICAL SOCIETY

Volume 77, Number 3, May 1971

\title{
ON INSEPARABLE GALOIS THEORY
}

\author{
BY STEPHEN U. CHASE ${ }^{1}$
}

Communicated by Alex Rosenberg, November 23, 1970

Throughout this note $k$ will be a field of characteristic $p \neq 0$, and $K$ will be a modular extension of $k$ [9]; i.e., a finite purely inseparable field extension of $k$ which is a tensor product, over $k$, of primitive extensions. We shall outline a Galois theory of modular field extensions which, for the special case where the exponent of $K / k$ is one, reduces to the well-known Galois correspondence of Jacobson [5, p. 186] between intermediate fields in the extension and restricted Lie subalgebras of $L(K / k)=\operatorname{Der}_{k}(K, K)$ which are also $K$-subspaces $(L(K / k)$ being the restricted Lie $k$-algebra and $K$-space of derivations of $K$ over $k$ ).

There have recently appeared in the literature a number of other approaches to inseparable Galois theory, in varying stages of development; see, e.g. Sweedler [8], [9], Shatz [7], Davis [2], Gerstenhaber and Zaromp [3]. Our treatment utilizes the Hopf algebraic techniques of [8].

1. Basic concepts. A cocommutative $k$-coalgebra $C[10$, p. 63] will be called a divided power coalgebra if $[C: k]$ is a power of $p$ and $C \approx C_{1}$ $\otimes_{k} \cdots \otimes_{k} C_{r}$, where each coalgebra $C_{i}$ is spanned by a sequence of divided powers [10, p. 268]. A divided power Hopf algebra is a Hopf $k$-algebra which is a divided power coalgebra. The $k$-space $P(C)$ of primitive elements of $C[10$, p. 199] is a restricted Lie $k$-algebra [4] if $C$ is a Hopf algebra, the Lie multiplication and $p$-power map in $P(C)$ being defined by the formulae $[x, y]=x y-y x$ and $x^{[p]}=x^{p}$ for $x, y$ in C.

TheOREM 1. There exists a divided power Hopf k-algebra $H(K / k)$ and a measuring $\omega_{K / k}: H(K / k) \otimes K \rightarrow K[10$, p. 138] with the following universal property. Given any measuring $\omega: C \otimes K \rightarrow K$, with $C$ a divided power $k$-coalgebra, there is a unique coalgebra map $f: C \rightarrow H(K / k)$ such that $\omega=\omega_{K / k}\left(f \otimes 1_{K}\right) . H(K / k)$ is uniquely determined by $K / k$ up to Hopf algebra isomorphism, and $[H(K / k): k]=[K: k]^{[K: k]}$. Moreover, there exists a restricted Lie algebra isomorphism $P(H(K / k)) \approx L(K / k)$,

A MS 1970 subject classifications. Primary 12F15; Secondary 16A24.

Key words and phrases. Inseparable field extensions, approximate automorphisms, higher derivations, Hopf algebras.

1 Supported in part by NSF GP-9395. The results of $\$ 1$ were presented to the Society on May 14, 1969. 
and if the exponent of $K / k$ is one there is a Hopf algebra isomorphism $H(K / k) \approx U_{r}(L(K / k))$, the restricted universal enveloping algebra of $L(K / k)$.

If $C$ is a subcoalgebra of $H(K / k)$, we shall denote by $K^{C}$ the subfield of fixed elements of $K$ under the measuring $\omega_{K / k}[10$, p. 202].

THEOREM 2. If $C$ is a divided power subcoalgebra of $H(K / k)$, then $K / K^{C}$ is modular [8, p. 274]. Conversely, if $k \subseteq F \subseteq K$ and $K / F$ is modular, then there is a unique divided power Hopf subalgebra $H(K / F)$ of $H(K / k)$ such that $K^{H(K / F)}=F$ and, if $C$ is as above, then $F \subseteq K^{C}$ if and only if $C \subseteq H(K / F)$. $[H(K / F): k]=[K: F]^{[K: k]}$.

In order to characterize those Hopf subalgebras of $H(K / k)$ of the form $H(K / F)$, with $F$ as above, we introduce a Hopf algebraic analogue of Jacobson's $K$-space structure on $L(K / k)$. Recall that, if $X$ is a cocommutative coalgebra and $Y$ is a commutative, cocommutative Hopf algebra, then $\operatorname{Coalg}_{k}(X, Y)$, the set of all coalgebra maps from $X$ to $Y$, is an abelian group, with composition law $*$ defined as in $[10$, p. 69].

Definition 3. A formal $K$-space is a commutative, cocommutative Hopf $k$-algebra $Y$, together with a map $K \times Y \rightarrow Y$ such that, for any $X$ as above, the induced map $K \times \operatorname{Coalg}_{k}(X, Y) \rightarrow \operatorname{Coalg}_{k}(X, Y)$ renders the abelian group $\operatorname{Coalg}_{k}(X, Y)$ a vector space over $K$. ( $Y$ itself is not a $K$-space; however, $P(Y)$ is.)

Now, if $C$ is a divided power $k$-coalgebra, we let $k=C(0) \subseteq C(1)$ $\subseteq \cdots \subseteq C(n) \subseteq \cdots$ be the natural (or coradical) filtration of $\bar{C}[10$, p. 185$]$, and denote by $\operatorname{gr}(C)$ the associated (strictly) graded coalgebra $[10$, p. 228]; the $k$-space of homogeneous elements of $\operatorname{gr}(C)$ of degree $n$ is $\operatorname{gr}(C)_{n}=C(n) / C(n-1)$. There exist natural $k$ space isomorphisms $\operatorname{gr}(C)_{1} \approx P(\operatorname{gr}(C)) \approx P(C)$. Finally, $\operatorname{gr}(C)$ possesses a unique $k$-algebra structure which renders it a commutative, cocommutative Hopf $k$-algebra.

THEOREM 4. There exists a unique map $K \times \operatorname{gr}(H(K / k))$ $\rightarrow \operatorname{gr}(H(K / k))$ rendering $\operatorname{gr}(H(K / k))$ a formal $K$-space such that the composite isomorphism $\operatorname{gr}(H(K / k))_{1} \approx P(H(K / k)) \approx L(K / k)$ is a $K$ space map.

If $H$ is a divided power Hopf subalgebra of $H(K / k)$, then the inclusion map $H \hookrightarrow H(K / k)$ induces an injection $\operatorname{gr}(H) \hookrightarrow \operatorname{gr}(H(K / k))$, and so we may identify $\operatorname{gr}(H)$ with a graded Hopf subalgebra of $\operatorname{gr}(H(K / k))$.

THEOREM 5. If $k \subseteq F \subseteq K$ and $K / F$ is modular, then $\operatorname{gr}(H(K / F))$ is a 
formal $K$-subspace of $\operatorname{gr}(H(K / k)$ ) (i.e., is closed under the action of $K$ introduced in Theorem 4). Conversely, if $H$ is a divided power Hopf subalgebra of $H(K / k)$ such that $\operatorname{gr}(H)$ is a formal $K$-subspace of $\operatorname{gr}(H(K / k))$, and $K$ is a tensor product over $F=K^{H}$ of primitive extensions of $F$ of equal exponent, then $H=H(K / F)$.

2. Regular Hopf algebras. One can deduce Jacobson's theorem $[5$, p. 186] as a special case of Theorem 5 . But in order to obtain a more complete and useful theory, it is desirable to examine more closely the various " $K$-actions" on Lie algebras and Hopf algebras discussed earlier. We begin with the Lie algebra case.

Definition 6. Let $A$ be an (associative) $k$-algebra containing $K$, and set $A_{K}^{+}=\{u$ in $A / u x-u x$ is in $K$ for all $x$ in $K\} . A_{K}^{+}$is a left $K$ subspace of $A$, and is a restricted Lie $k$-algebra under the operations $[u, v]=u v-v u$ and $u^{[p]}=u^{p}$ for $u, v$ in $A_{K}^{+}$.

THEOREM 7 [6], [1]. The following are equivalent for any restricted Lie $k$-algebra and $K$-space $L$ :

(a) There exists an associative k-algebra $A$ containing $K$, and $a$ restricted Lie k-algebra and $K$-space injection $j: L \hookrightarrow A_{K}^{+}$.

(b) There exists a restricted Lie k-algebra map $\delta: L \rightarrow L(K / k)$ such that (if $u(x)=\delta(u)(x)$ for $u$ in $L$ and $x$ in $K)[\alpha u, \beta v]=\alpha u(\beta) v-\beta v(\alpha) u$ $+\alpha \beta[u, v]$ and $(\alpha u)^{[p]}=\alpha^{p} u^{[p]}+u(\alpha)^{p-1} u$ for $u, v$ in L and $\alpha, \beta$ in $K$.

The map $\delta$ above is uniquely determined by $L$. We shall say that $L$ is $K$-regular if it satisfies the conditions of Theorem 7 ; such Lie algebras were first considered by Hochschild in [4].

We introduce analogous notions for Hopf algebras. Let $A$ be a $k$ algebra containing $K$, and $H$ be a divided power Hopf $k$-algebra. A $k$-algebra map $\varphi: H \rightarrow A$ will be called admissible if, for any $u$ in $H$ and $x$ in $K, \sum_{(u)} f\left(u_{(1)}\right) x f\left(\lambda\left(u_{(2)}\right)\right)$ is also in $K$, where $\lambda$ and $\Delta$ are the antipode $[10$, p. 71$]$ and diagonal maps of $H$, respectively, and $\Delta(u)$ $=\sum_{(u)} u_{(1)} \otimes u_{(2)}$. Finally, the length of a maximal sequence of divided powers of $H$ is a power of $p$; this power is called the exponent of $H$.

THEOREM 8. For any natural number e and k-algebra $A$ containing $K$, with $[A: k]<\infty$, there exists a divided power Hopf $k$-algebra $h_{K}^{e}(A)$ of exponent $e$ and an admissible map $\zeta_{A}: h_{K}^{e}(A) \rightarrow A$ with the following universal property: Given any divided power Hopf k-algebra $H$ of exponent $\leqq e$ and admissible map $\varphi: H \rightarrow A$, there is a unique Hopf $k$ algebra map $f: H \rightarrow h_{K}^{e}(A)$ such that $\zeta_{A} f=\varphi$. $h_{K}^{e}(A)$ is unique up to Hopf algebra isomorphism; moreover-

(a) $P\left(h_{K}^{e}(A)\right) \approx A_{K}^{+}$as restricted Lie k-algebras. 
(b) $h_{K}^{1}(A) \approx U_{r}\left(A_{K}^{+}\right)$as Hopf k-algebras.

(c) There exists a unique formal $K$-space structure on $\operatorname{gr}\left(h_{K}^{e}(A)\right)$ such that the composite isomorphism $\operatorname{gr}\left(h_{K}^{e}(A)\right)_{1} \approx P\left(h_{K}^{e}(A)\right) \approx A_{K}^{+}$is a $K$-space map.

(d) If $\left\{1, u_{1}, u_{2}, \cdots, u_{n}\right\}$ is a sequence of divided powers of $h_{\mathrm{K}}^{e}(A)$ and $\alpha$ is in $K$, then there is a unique sequence $\left\{1, \alpha \circ u_{1}, \alpha \circ u_{2}\right.$, $\left.\cdots, \alpha \circ u_{n}\right\}$ of divided powers of $h_{K}^{e}(A)$ such that $\zeta_{A}\left(\alpha \circ u_{i}\right)=\alpha^{i} \zeta_{A}\left(u_{i}\right)$ for $i \leqq n$.

Definition 9. Let $H$ be a divided power Hopf $k$-algebra, with $\operatorname{gr}(H)$ a formal $K$-space. $H$ is called $K$-regular if there exists a $k$ algebra $A$ containing $K$, with $[A: k]<\infty$, and a Hopf $k$-algebra injection $j: H \leftrightharpoons h_{K}^{e}(A)$ for some natural number $e$, such that-

(a) $\operatorname{gr}(j): \operatorname{gr}(H) \hookrightarrow \operatorname{gr}\left(h_{K}^{e}(A)\right)$ is a map of formal $K$-spaces.

(b) If $\alpha$ is in $K$ and $\left\{1, u_{1}, \cdots, u_{n}\right\}$ is a sequence of divided powers of $\operatorname{Im}(j)$, then $\alpha \circ u_{i}$ is likewise in $\operatorname{Im}(j)$ whenever $p \mid i$.

If $L$ is a restricted Lie $k$-algebra and $K$-space of finite dimension, then one obtains easily a unique formal $K$-space structure on $\operatorname{gr}\left(U_{r}(L)\right)$ such that the composite isomorphism $L \approx P\left(U_{r}(L)\right)$ $\approx P\left(\operatorname{gr}\left(U_{r}(L)\right)\right) \approx \operatorname{gr}\left(U_{r}(L)\right)_{1}$ is a $K$-space map.

THeOREM 10. If $L$ is as above, then $U_{r}(L)$ is a K-regular Hopf algebra if and only if $L$ is a $K$-regular restricted Lie algebra.

3. The fundamental theorem and an application. Our Galois correspondence now assumes the following form.

THEOREM 11. If $K / k$ is modular, then there exists a one-to-one latticeinverting correspondence between the fields $F$, with $k \subseteq F \subseteq K$ and $K / F$ modular, and the $K$-regular Hopf subalgebras $H$ of $H(K / k)$. The correspondence is given by the operations $H \rightarrow K^{H}, F \rightarrow H(K / F)$.

The theorem below is a consequence of the Galois theory developed here, together with some of the results and techniques of [10]. If $A$ is a $k$-algebra, let $A[t]$ be the ring of "truncated" polynomials over $A$ in the indeterminate $t$, subject only to the relation $t^{m}=0$ for some natural number $m$. An approximate automorphism of $K / k(\bmod$ degree $m)$ is a $k[t]$-algebra automorphism $\sigma$ of $K[t]$ such that $\sigma(x)=x(\bmod t K[t])$ for all $x$ in $K \subseteq K[t]$ [3]. If $A$ is a $k$-algebra containing $K$, then an approximate automorphism $\sigma$ of $K / k$ is called $A$-inner if there is an invertible element $u$ of $A[t]$ such that $\sigma(x)$ $=u x u^{-1}$ for all $x$ in $K \subseteq A \subseteq A[t]$.

TheOREM 12. Let $K$ be a finite extension field of $k$, and $A$ be $a k$ algebra containing $K$, with $[A: k]<\infty$. Assume that the subfield of $K$ 
of elements left fixed by all A-inner approximate automorphisms of $K / k$ is precisely $k$ (i.e., if $x$ is in $K$, then $\sigma(x)=x$ for every $A$-inner approximate automorphism $\sigma$ of $K / k$ if and only if $x$ is in $k)$. If $B=\{a$ in $A / a x=x a$ for all $x$ in $K\}$ is the centralizer of $K$ in $A$, then $A$ is a free left and right $B$-module of rank $[K: k]$, and the $\operatorname{map} \varphi: A \otimes_{k} K \rightarrow \operatorname{End}_{B}(A)$ is an isomorphism of $K$-algebras, where $\varphi(a \otimes x) u=a u x$ for $a, u$ in $A$ and $x$ in $K$, and $A$ is viewed as a right $B$-module.

In particular, $A$ is a "form" of the $K$-algebra of $n \times n$ matrices over $B$, where $n=[K: k]$.

\section{REFERENCES}

1. G. Angwin, On regular restricted Lie algebras (unpublished).

2. R. L. Davis, $A$ Galois theory for a class of purely inseparable exponent two field extensions, Bull. Amer. Math. Soc. 75 (1969), 1001-1004. MR 39 \#5524.

3. M. Gerstenhaber and A. Zaromp, On the Galois theory of purely inseparable field extensions, Bull. Amer. Math. Soc. 76 (1970), 1011-1014.

4. G. Hochschild, Simple algebras with purely inseparable splitting fields of exponent one, Trans. Amer. Math. Soc. 79 (1955), 477-489. MR 17, 61.

5. N. Jacobson, Lectures in abstract algebra. Vol. III: Theory of fields and Galois theory, Van Nostrand, Princeton, N. J., 1964. MR 30 \#3087.

6. G. S. Rinehart, Differential forms on general commutative algebras, Trans. Amer. Math. Soc. 108 (1963), 195-222. MR 27 \#4850.

7. S. Shatz, Galois theory, Lecture Notes in Math., no. 86, Springer-Verlag, Berlin, 1969, pp. 146-158.

8. M. E. Sweedler, The Hopf algebra of an algebra applied to field theory, J. Algebra 8 (1968), 262-276. MR 36 \#5105.

9. - Structure of inseparable extensions, Ann. of Math. (2) 87 (1968), 401410. MR $36 \# 6391$.

10. - Hopf algebras, Math. Lecture Note Series, Benjamin, New York, 1969. MR $40 \# 5705$.

Cornell University, Ithaca, New York 14850 